\section{A pharmacological approach to first aid treatment for snakebite}

\author{
Megan E Saul ${ }^{1}$, Paul A Thomas ${ }^{2}$, Peter J Dosen ${ }^{3}$, Geoffrey K Isbister ${ }^{4,5}$, \\ Margaret A O'Leary ${ }^{5}$, Ian M Whyte ${ }^{4,5}$, Sally A McFadden ${ }^{6}$ \& \\ Dirk F van Helden ${ }^{3}$

\begin{abstract}
Snake venom toxins first transit the lymphatic system before entering the bloodstream. Ointment containing a nitric oxide donor, which impedes the intrinsic lymphatic pump, prolonged lymph transit time in rats and humans and also increased rat survival time after injection of venom. This pharmacological approach should give snakebite victims more time to obtain medical care and antivenom treatment.
\end{abstract}

Each year snakebite accounts for some 100,000 deaths and 400,000 amputations worldwide ${ }^{1-3}$. Many snake venoms contain large toxin molecules that cannot enter the bloodstream directly but are absorbed and transported by lymphatic vessels before entering veins near the heart ${ }^{4}$. This has led to mechanical first aid procedures for snakebite, including pressure bandage with immobilization $(\mathrm{PBI})^{5}$ and local pressure pad compression ${ }^{6,7}$. These techniques aim to delay toxins from reaching the circulation by impeding lymphatic transport without compromising arterial blood flow.

Three primary mechanisms influence lymph flow: fluid entry through endothelial valves into the end lymphatics ${ }^{8}$ (absorption), propulsion of lymph by external compression ${ }^{8}$ and intrinsic pumping mediated by smooth muscle contractions in the lymphatic vessel wall ${ }^{9}$. Notably, the intrinsic pump is the dominant propulsive mechanism in humans under resting conditions ${ }^{10}$. As nitric oxide (NO) impedes the intrinsic pump ${ }^{11}$, we hypothesized that NO-releasing agents applied topically would slow lymphatic transit time and entry of the venom into the circulation, delaying onset of systemic toxicity.

We tested an ointment (GTNO) containing the NO donor glyceryl trinitrate ( $0.2 \% \mathrm{wt} / \mathrm{wt}$; Rectogesic, Care Pharmaceuticals; commercially available) in human volunteers (six male, nine female; age 20-65 years; Supplementary Methods). We simulated snakebite using a validated method $^{12}$ with $50 \mu \mathrm{l}$ of sterile radiolabeled colloid injected subcutaneously in the dorsum of the foot, with the subject instructed to remain still for the entire procedure. Lymphatic transit times were measured from injection to the first measurement of activity in groin lymph nodes using a radiation-detecting camera. On another day, the subjects underwent the same procedure, but within $1 \mathrm{~min}$ of colloid injection had $15 \mathrm{~g}$ of GTNO topically applied thickly in a circle ( $\sim 5 \mathrm{~cm}$ diameter) proximal to the injection site. GTNO application caused marked slowing in foot-to-groin lymph transit time in each subject from $13 \mathrm{~min}$ (range 4-81 min) without GTNO to $54 \mathrm{~min}$ (range 6.5-162 min) with GTNO, a $344 \pm 52 \%$ increase (mean \pm s.e.m.; $n=15 ; P<0.0001$; Fig. 1).

We also undertook lymphatic transit measurements in rats anesthetized with urethane ( 1.5 g per kg body weight intraperitoneally) and maintained at $37^{\circ} \mathrm{C}$. Groin lymphatic vessels were surgically exposed (Supplementary Fig. 1). We measured foot-to-groin lymph transit times visually through a microscope in the presence of GTNO or a base ointment, with ointment applied over the entire leg within $20 \mathrm{~s}$ after hind foot injection of marker dye (India ink ${ }^{13}$ ). Transit time with GTNO treatment was threefold greater compared to control treatment ( $9.4 \pm 0.8 \mathrm{~min}, n=13$ with GTNO versus $3.2 \pm 0.2 \mathrm{~min}, n=16$ without GTNO; $P<0.0001)$. This outcome was similar to that observed in humans (Fig. 1). There was no significant difference $(P=0.12)$ between the effects of GTNO $(0.2 \% \mathrm{wt} / \mathrm{wt})$, and another NO donor, $10 \mathrm{mM}$ sodium nitroprusside (SNP; 0.3\% wt/wt in saline with $1 \%$ DMSO). These two agents increased hind limb lymphatic transit times relative to vehicle by $298 \%$ and $313 \%$, respectively, indicating that a second NO donor has the same effect. There was no additional effect of $100 \mathrm{mM}$ compared to $10 \mathrm{mM}$ SNP (hind limb lymph transit relative to vehicle was slowed by $304 \%(n=7,100 \mathrm{mM} \mathrm{SNP} ; P<0.0002)$ and $313 \%$ ( $n=11,10 \mathrm{mM} \mathrm{SNP}, n=13$ vehicle; $P<0.0002)$.

GTNO action was likely to be on the intrinsic pump rather than on absorption of the tracer or external compression by skeletal muscle. GTNO application to only the rat leg above the ankle, away from the foot injection site, gave a transit time of $11.5 \pm 0.9 \mathrm{~min}(n=8)$, which was not substantially different to GTNO applied over both the foot and leg, arguing against an effect of GTNO on absorption. The rats were anesthetized, making skeletal muscle involvement unlikely. Furthermore, stimulation of hind limb muscle contraction at $2 \mathrm{~Hz}$ did not alter normal lymph transit time as assessed using ink injection $(3.1 \pm 0.2 \mathrm{~min}, n=12$; $P=0.85)$. Notably, GTNO application increased lymph transit time even when hind limb muscle contraction was stimulated in this way (4.6 \pm $0.4 \mathrm{~min}, n=12 ; P<0.005)$ compared to without GTNO $(3.1 \pm 0.2 \mathrm{~min}$, $n=12$ ). These findings indicate that topical administration of NO donors in vivo inhibits smooth muscle contractions underlying the intrinsic lymphatic pump, as has been observed in vitro ${ }^{11}$.

We examined the efficacy of GTNO in inhibiting the effects of a snake venom (Pseudonaja textilis, Eastern Brown snake), injected into the hind foot of anesthetized nonrecovery rats, by measuring changes in the time to respiratory arrest, respiratory rate and optical absorbance of plasma. We applied ointment (GTNO or control) over the entire hind limb within $20 \mathrm{~s}$ of venom injection (dose range 0.4-1.2 mg per kg body weight). GTNO significantly increased time to respiratory arrest from $65 \pm 4 \mathrm{~min}$ in control rats to $96 \pm 6 \mathrm{~min}$ in

${ }^{1}$ Department of Nuclear Medicine, John Hunter Hospital, New Lambton, New South Wales, Australia. ${ }^{2}$ Department of Nuclear Medicine, Royal Brisbane and Women's Hospital, Herston, Queensland, Australia. ${ }^{3}$ School of Biomedical Sciences \& Pharmacy, University of Newcastle, Callaghan, New South Wales, Australia. ${ }^{4}$ School of Medicine and Public Health, University of Newcastle, Callaghan, New South Wales, Australia. ${ }^{5}$ Department of Clinical Toxicology and Pharmacology, Calvary Mater Newcastle, Waratah, New South Wales, Australia. ${ }^{6}$ School of Psychology, University of Newcastle, Callaghan, New South Wales, Australia. Correspondence should be addressed to D.F.v.H. (dirk.vanhelden@newcastle.edu.au).

Received 11 February; accepted 19 April; published online 26 June 2011; doi:10.1038/nm.2382 


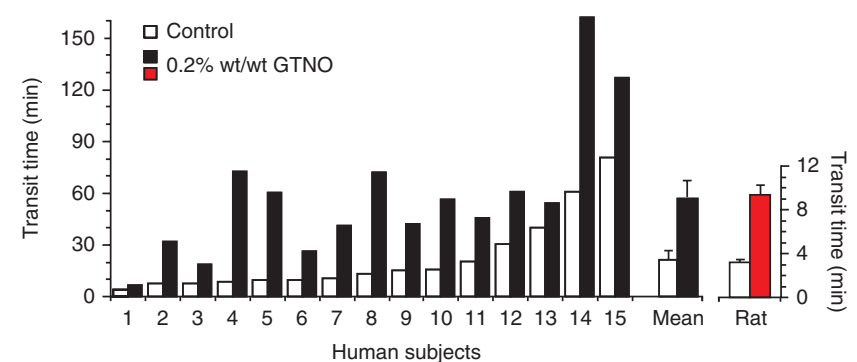

Figure 1 Effects of topical application of GTNO on lymphatic transit times. Foot-to-groin lymphatic transit times in each of 15 human subjects with and without GTNO treatment and the mean \pm s.e.m. transit time ( $n=15$; left). Foot-to-groin lymphatic transit times in rats (mean \pm s.e.m.) treated with GTNO ( $n=16$ ) or control base ointment (right; $n=13$; right-hand $y$ axis applies to the rat data only).

GTNO-treated rats $(P<0.001$; log-rank test; Fig. 2a) and attenuated the decrease in respiratory rate in GTNO-treated rats versus control rats (Fig. 2b). GTNO also reduced the increase in optical absorbance (reflecting attenuation of venom-induced hemolysis and clotting with fibrin strand formation); after $15 \mathrm{~min}$, optical absorbance increased rapidly to 0.4 relative units in the plasma of control rats, compared to half that value in the plasma of GTNO-treated rats (Fig. 2c). GTNO did not have a specific effect at the site of venom injection, as the effects of GTNO administration did not differ significantly when we applied it above the ankle away from the foot $(n=3)$ compared to over both the foot and leg $(n=9 ; P=0.47)$.

The finding that GTNO caused a 6-min increase in lymph transit time ( 9.4 versus $3.2 \mathrm{~min}$ ) but a $31-\mathrm{min}$ increase in time to venominduced respiratory arrest (96 versus $65 \mathrm{~min}$ ) indicates that a small effect on venom entry into the system results in a greater delay in venom-induced effects. The magnitude of this delay will depend on the input system, the nature of the toxin and its sites of action. Lymphatic delivery is best represented as a zero-order input process, for which transit time is inversely proportional to rate of delivery, whereas distribution of toxins to target sites (for example, nerves and muscles) and elimination of toxins from the body are first-order processes $^{14,15}$. Delaying venom transportation through the lymphatics is likely to decrease the peak venom concentration in blood and therefore venom-induced effects. However, the relationship between changes in lymphatic transit time and delays in different venom-induced effects will be complex, as evidenced by the varying effects that GTNO had on time to respiratory arrest, respiratory rate and plasma absorbance over time (Fig. 2).

A mechanical PBI-like first aid procedure (pressure cuff application at $50 \mathrm{~mm} \mathrm{Hg}$ to the rat hind limb) prevented respiratory arrest for the $2-3 \mathrm{~h}$ period that the cuff was present $(n=4)$, consistent with previous findings ${ }^{5}$. This technique effectively occludes rather than slows lymph flow. However, application of PBI requires considerable skill, with untrained and trained personnel respectively achieving only $14 \%$ and $50 \%$ success rates ${ }^{16}$ and it fails when there is movement ${ }^{12}$. PBI is currently recommended in Australia but not in other countries, as snakes in Australia tend to cause minimal local tissue damage compared to the cytotoxic venoms of many other snakes, for which delaying systemic entry of the venom toxin using PBI would increase local tissue damage. However, mechanical and pharmacological approaches that impede lymphatic flow need to be considered for use against a wider range of snakes, as PBI has been reported to be a useful first aid against simulated rattlesnake (Crotalus atrox) bites in pigs $^{17}$, a venom that causes severe local tissue effects.
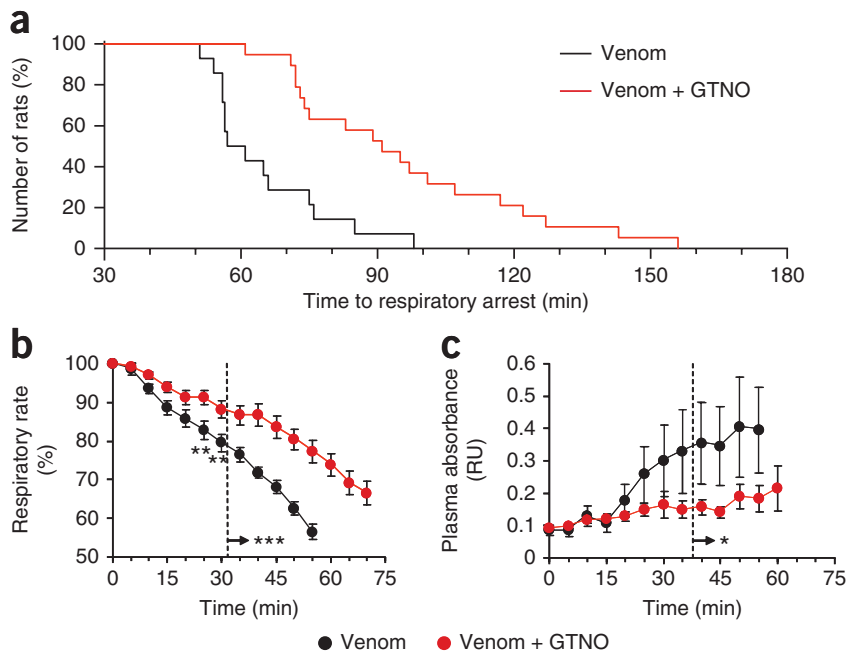

Figure 2 Effects of GTNO treatment on venom actions in anesthetized rats. (a) Kaplan-Meier plot showing GTNO effects on the time to respiratory arrest after subcutaneous venom injection in the rat hind foot ( $n=14$ control; $n=19$ GTNO). (b) Respiratory rate over time (mean \pm s.e.m.). (c) 405-nm optical absorbance (relative units, RU) of plasma over time (mean \pm s.e.m.) in control- $(n=6)$ and GTNO-treated rats $(n=7)$. Data comparison was made using two-way repeated-measures analysis of variance with Holm-Sidak post hoc tests $\left({ }^{*} P<0.05,{ }^{* *} P<0.01\right.$, $* * * P<0.001)$. Comparisons between venom + base ointment and venom + GTNO to the right of the dashed lines in $\mathbf{b}$ and $\mathbf{c}$ are all significantly different.

Our findings indicate that a topical pharmacological approach, using an NO donor, inhibits lymphatic transit in humans and rats. These results point to a new method of snakebite first aid that may also be useful for bites to the torso or head. Of note, our lymph transit measurements in rats indicated a benefit of GTNO even with muscle movement, whereas the PBI approach fails when humans move ${ }^{12}$. GTNO and PBI may together give considerably more benefit with movement, as GTNO targets the intrinsic pump, which can generate lymphatic pressures of $100 \mathrm{~mm} \mathrm{Hg}^{10}$, well above the recommended bandage pressure range of $55-70 \mathrm{~mm} \mathrm{Hg}^{12}$.

The human studies were approved by the Hunter New England Area Health Human Research Ethics Committee and informed consent was obtained from all subjects. The rat studies were approved by the University of Newcastle Animal Care and Ethics Committee.

Note: Supplementary information is available on the Nature Medicine website.

\section{ACKNOWLEDGMENTS}

We sincerely thank L. Milward and D. Laver for critical evaluation of the manuscript, the National Health and Medical Research Council of Australia and Hunter Medical Research Institute for funding support and J. Weigel from The Australian Reptile Park for the donation of snake venom.

\section{AUTHOR CONTRIBUTIONS}

M.E.S. conducted the human experiments. P.A.T. supervised the human experiments. P.J.D. conducted the rat experiments. G.K.I. provided expertise on rat experiments, supervised assays and assisted in drafting the manuscript and statistical advice. M.A.O. undertook the absorbance assays. I.M.W. provided input on the human experiments. S.A.M. undertook the statistical analysis and figure presentations. D.F.v.H. conceived of and directed the overall project, supervised the rat experiments, analyzed data and drafted the manuscript.

\section{COMPETING FINANCIAL INTERESTS}

The authors declare competing financial interests: details accompany the full-text HTML version of the paper at http://www.nature.com/naturemedicine/. 


\section{BRIEF COMMUNICATIONS}

Published online at http://www.nature.com/naturemedicine/.

Reprints and permissions information is available online at http://www.nature.com/ reprints/index.html.

1. Chippaux, J.P. Bull. World Health Organ. 76, 515-524 (1998).

2. Gilchrist, J. in World Report on Child Injury Prevention (eds. Peden, M. et al.) Chapter 6, Box 6.1 (World Health Organization, Geneva, 2008).

3. Kasturiratne, A. et al. PLoS Med. 5, e218 (2008).

4. Barnes, J.M. \& Trueta, J. Lancet 237, 623-626 (1941).

5. Sutherland, S.K., Coulter, A.R. \& Harris, R.D. Wilderness Environ. Med. 16, 164-167 (2005)

6. Anker, R.L., Straffon, W.G. \& Loiselle, D.S. Med. J. Aust. 1, 103 (1982)

7. Hack, J.B. et al. J. Med. Toxicol. 6, 207-211 (2010).
8. Schmid-Schönbein, G.W. Physiol. Rev. 70, 987-1028 (1990).

9. Aukland, K. Acta Physiol. Scand. 185, 171-180 (2005).

10. Olszewski, W.L. \& Engeset, A. Am. J. Physiol. 239, H775-H783 (1980).

11. Yokoyama, S. \& Ohhashi, T. Am. J. Physiol. 264, H1460-H1464 (1993)

12. Howarth, D.M., Southee, A.E. \& Whyte, I.M. Med. J. Aust. 161, 695-700 (1994).

13. Marco, A.J. et al. Lab. Anim. 26, 200-205 (1992)

14. Rivière, G., Choumet, V., Saliou, B., Debray, M. \& Bon, C. J. Pharmacol. Exp. Ther 285, 490-495 (1998).

15. Krifi, M.N. et al. Toxicon 45, 187-198 (2005)

16. Canale, E., Isbister, G.K. \& Currie, B.J. Emerg. Med. Australas. 21, 184-190 (2009)

17. Meggs, W.J., Courtney, C., O'Rourke, D. \& Brewer, K.L. Clin. Toxicol. (Phila.) 48 61-63 (2010). 